\title{
Latitude dependence of long-term geomagnetic activity and its solar wind drivers
}

\author{
M. Myllys ${ }^{1}$, N. Partamies ${ }^{2,3}$, and L. Juusola ${ }^{2}$ \\ ${ }^{1}$ Department of Physics, University of Helsinki, Helsinki, P.O. Box 64, Finland \\ ${ }^{2}$ Finnish Meteorological Institute, Erik Palmenin aukio 1, 00560 Helsinki, Finland \\ ${ }^{3}$ Department of Arctic Geophysics, The University Centre in Svalbard, Longyearbyen, Norway \\ Correspondence to: M. Myllys (minna.myllys@helsinki.fi)
}

Received: 20 October 2014 - Revised: 23 April 2015 - Accepted: 24 April 2015 - Published: 28 May 2015

\begin{abstract}
To validate the usage of global indices in studies of geomagnetic activity, we have examined the latitude dependence of geomagnetic variations in Fennoscandia and Svalbard from 1994 to 2010. Daily standard deviation (SD) values of the horizontal magnetic field have been used as a measure of the ground magnetic disturbance level. We found that the timing of the geomagnetic minimum depends on the latitude region: corresponding to the minimum of sunspot cycle 22 (in 1996), the geomagnetic minimum occurred between the geomagnetic latitudes $57-61^{\circ}$ in 1996 and at the latitudes $64-67^{\circ}$ in 1997 , which are the average auroral oval latitudes. During sunspot cycle 23, all latitude regions experienced the minimum in 2009, a year after the sunspot minimum. These timing differences are due to the latitude dependence of the 10 s daily SD on the different solar wind drivers. In the latitude region of $64-67^{\circ}$, the impact of the high-speed solar wind streams (HSSs) on the geomagnetic activity is the most pronounced compared to the other latitude groups, while in the latitude region of $57-61^{\circ}$, the importance of the coronal mass ejections (CMEs) dominates. The geomagnetic activity maxima during ascending solar cycle phases are typically caused by CME activity and occur especially in the oval and sub-auroral regions. The strongest geomagnetic activity occurs during the descending solar cycle phases due to a mixture of CME and HSS activity. Closer to the solar minimum, less severe geomagnetic activity is driven by HSSs and mainly visible in the poleward part of the auroral region. According to our study, however, the timing of the geomagnetic activity minima (and maxima) in different latitude bands is different, due to the relative importance of different solar wind drivers at different latitudes.
\end{abstract}

Keywords. Geomagnetism and paleomagnetism (time variations diurnal to secular)

\section{Introduction}

Solar activity varies in about 11-year cycles (Schove, 1955) and is the driving force for long-term geomagnetic variations. Solar wind properties determine the reconnection rate at the boundary of the magnetosphere. Because the magnetosphere is coupled to the ionosphere (Wolf, 1975), solar wind driving has an impact on the ionospheric current systems, which, in turn, cause geomagnetic disturbances. The occurrence of geomagnetic disturbances is then also subject to 11-year periods, which are less obvious and are delayed by about $1-$ 2 years compared to the sunspot cycle (Fraser-Smith, 1972; Pulkkinen et al., 2011).

The solar cycle evolution of geomagnetic activity often consists of two peaks during the solar maximum and in the declining phase (Gonzalez et al., 1990; Echer et al., 2004). The coronal mass ejections (CMEs) and high-speed solar wind streams (HSSs) drive the most severe geomagnetic variations. The relative importance of these two drivers varies according to the phase of the sunspot cycle. The occurrence rate of CMEs increases with the enhanced solar activity (Gopalswamy et al., 2004), while the number of HSSs peaks during the declining phase of the sunspot cycle (Tanskanen et al., 2005). The first geomagnetic activity peak during the sunspot maximum is often related to an enhanced occurrence rate of CMEs, while the second peak mostly relates to the increased high-speed stream frequency later in the same spot cycle (Gonzalez et al., 1990; Richardson et al., 2001). 
Table 1. Corrected geomagnetic coordinates of the IMAGE magnetometer stations used in this study. The stations are listed from north to south, and the station groups (Region 1, Region 2 and Region 3) have been separated by horizontal lines.

\begin{tabular}{llr}
\hline Station & CGM latitude & CGM longitude \\
\hline NAL, Ny-Ålesund & 75.25 & 112.08 \\
LYR, Longyearbyen & 75.12 & 113.00 \\
HOR, Hornsund & 74.13 & 109.59 \\
HOP, Hopen Island & 73.06 & 115.10 \\
\hline SOR, Sørøya & 67.34 & 106.17 \\
KEV, Kevo & 66.32 & 109.24 \\
TRO, Troms $\varnothing$ & 66.64 & 102.90 \\
MAS, Masi & 66.18 & 106.42 \\
KIL, Kilpisjärvi & 65.94 & 103.80 \\
MUO, Muonio & 64.72 & 105.22 \\
LOZ, Lovozero & 64.23 & 114.49 \\
PEL, Pello & 63.55 & 104.92 \\
\hline OUJ, Oulujärvi & 60.99 & 106.14 \\
HAN, Hankasalmi & 58.69 & 104.54 \\
NUR, Nurmijärvi & 56.89 & 102.18 \\
\hline
\end{tabular}

The geomagnetic activity cycle is often described using geomagnetic indices, such as the aa index (Mayaud, 1972; Lockwood et al., 2013a). The aa is a range index based on the variations in the horizontal components of Earth's magnetic field detected by two antipodal magnetometer stations which are located in southern England and in Australia. The first geomagnetic activity peak, as described by aa, experiences a delay of up to about 1 year with respect to the sunspot number, and the second peak comes with a time lag of around 2-4 years (Echer et al., 2004). Sometimes, the geomagnetic activity maximum consists of multiple peaks as a combination of different driver mechanisms (Echer et al., 2004).

The solar-wind-magnetosphere coupling and the energy transfer into the magnetosphere have been widely studied using different coupling functions. The pioneering coupling function is Akasofu's epsilon parameter (Akasofu, 1981). This parameter connects the interplanetary magnetic field $(\boldsymbol{B})$ and the solar wind speed $(V)$, which have been found to correlate well with the geomagnetic activity (Lockwood et al., 2013a, b; Finch et al., 2008). Other commonly used coupling functions also consist of $V$ and $\boldsymbol{B}$ in different combinations of their multiplications and exponents of $V$. Some of the most widely used coupling functions have been listed by Finch and Lockwood (2007) and Newell et al. (2008) (Table 1 in both papers), and a new coupling function was recently published by Tenfjord and Østgaard (2013).

There are numerous types of geomagnetic indices for various purposes (Lockwood et al., 2013a). Some of the indices, such as aa, are intended to measure the geomagnetic activity in general, and others (Dst, AL and AU; Rostoker, 1972) measure a certain magnetospheric phenomena, such as the ring current enhancement or auroral electrojets. All the indices react differently to different solar wind drivers. The dependence of the interplanetary magnetic field (IMF) magnitude and solar wind speed of each geomagnetic index is ultimately determined by the instrumentation and instrument calibration procedures, the universal and magnetic local times, and the locations of the magnetometer stations (Lockwood et al., 2013a). The location of the station is influenced by different combinations of current systems, which respond differently to solar wind drivers (Lockwood et al., 2013b). Thus, the geomagnetic index should always be chosen carefully to support the topic of the study.

Finch et al. (2008) developed a geomagnetic index based on the monthly standard deviations of the $\boldsymbol{H}$ component of the geomagnetic field and studied the latitudinal correlation of the index with IMF and $V$. The use of the standard deviation removes the need to define the baseline for quiet days. The new index can be used to explore the geomagnetic variations at individual magnetometer stations. Based on the results of Finch et al. (2008), geomagnetic activity at most latitudes correlates best with the IMF magnitude, but the auroral region (60-82 ${ }^{\circ}$ geomagnetic latitudes), and especially the auroral midnight sector, shows strong dependency on $V$, which is assumed to be due to auroral electrojets and the substorm current wedge.

The solar cycle evolution of the CME occurrence rate during solar cycle 23 has been studied by several authors (Gopalswamy et al., 2004; Mittal and Narain, 2009). The cycle started in 1996, the sunspot maximum was reached in 2000 and the minimum in 2008. During the sunspot maximum year 2000, over 1500 CMEs were detected from LASCO (the Large Angle and Spectrometric Coronograph; Brueckner et al., 1995) data. This was significantly more than during the previous year (1999), when the number of CMEs was below 1000 (Gopalswamy et al., 2004). It was also the maximum number of Earth-directed CMEs in 1996-2006 (Mittal and Narain, 2009). The smallest number of halo CMEs $(<20)$ was observed in 1996.

During the past two solar cycles, substorms were more intense and frequent during the declining phases (in 1994 and 2003) of the sunspot cycles (Tanskanen et al., 2011). This is mainly due to the enhanced HSS rate (Tanskanen et al., 2005). At the solar activity minima in 1996-1997, the number of storm main phases was $<125$ and that of substorm expansion phases was < 3500; in 2008-2009 the number of main phases was $<50$ and that of expansion phases was $<3000$ (Partamies et al., 2013). During both minima magnetic activity measured in numbers of storms and substorms was low compared to the other years during solar cycle 23 , when the number of storm main phases was typically $>3500$ and that of the substorm expansion phases was $>125$. A couple of years after the solar activity maximum (2002-2003), the number of storms showed an increase. The substorms and storm phases were selected using the automatic substorm identification procedure described by Juusola et al. (2011). 


\section{IMAGE Magnetometer Network}

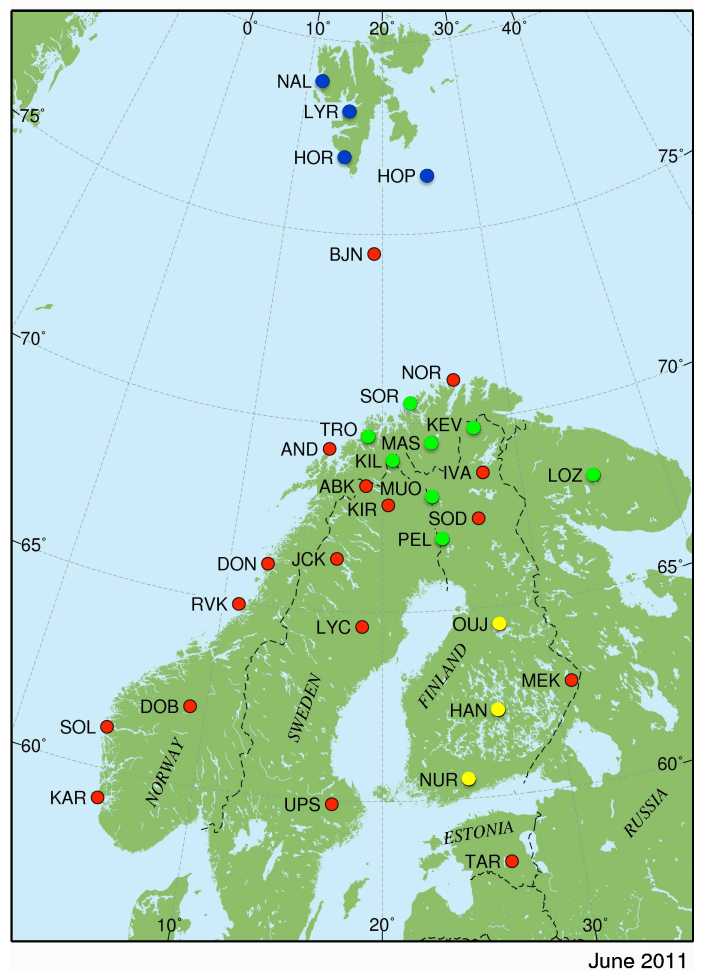

Figure 1. IMAGE magnetometer stations in 2011. The blue stations belong to Region 1, the green stations to Region 2 and Region 3 is marked with yellow.

In general, the storm occurrence rate stayed quite stable in 1995-2009, except during the deep and exceptionally long solar minimum in 2006-2009 (Partamies et al., 2013).

The location and the conductivity of the auroral oval changes during magnetic activity (Vickrey et al., 1981; Meng, 1984) caused latitudinal variations in ionospheric current systems (Solovyev et al. (2009); Pulkkinen et al. (2011) and references therein).The auroral electrojet currents became significantly weaker during 2008, and the electrojet moved poleward by almost $2^{\circ}$, indicating that the polar cap was smaller during the deep solar minimum in 2008-2010 than it usually is during solar minimum conditions (Pulkkinen et al., 2011; Imber et al., 2013).

The time derivative of the geomagnetic field $\mathrm{d} \boldsymbol{B} / \mathrm{d} t$, and especially its horizontal component $\mathrm{d} \boldsymbol{H} / \mathrm{d} t$, has been commonly used as an indicator of the geomagnetic variations. For example, diurnal and seasonal variations in geomagnetic activity have been examined as directional distributions of $\mathrm{d} \boldsymbol{H} / \mathrm{d} t$ (Viljanen et al., 2001; Falayi and Rabiu, 2013). The latitude behaviour of $\mathrm{d} \boldsymbol{H} / \mathrm{d} t$ in 2003-2005 differed systematically between different geomagnetic latitude regions (Watermann and Gleisner, 2009). The largest time derivatives can be found in the auroral zone during storm conditions. The amplitudes of $\mathrm{d} \boldsymbol{H} / \mathrm{d} t$ in the polar cap are always smaller than those in the auroral zone and the smallest values can be found at the sub-auroral latitudes under quiet and moderately disturbed conditions. During severe storms the time derivatives at the sub-auroral latitudes may exceed the values in the auroral zone.

In this paper, we use daily standard deviation values (SD) as a measure of the geomagnetic disturbance level. Previously, SD has been used to determine the general risk to the UK power grid from rapid magnetic variations (Beamish et al., 2002).

According to Finch et al. (2008), the activity in the auroral region (geographic latitudes $60-82^{\circ}$ ) correlates best with the coupling functions which include the solar wind speed. In the study, the oval region covers almost the whole IMAGE (International Monitor for Auroral Geomagnetic Effects) magnetometer network. Because of the coarse latitude resolution, the study does not give very good images of the geomagnetic variations in the high-latitude region. We divided the groundbased magnetic field SD values into three latitude bands: 73 $75,64-67$ and $57-61^{\circ}$. These regions correspond to the average poleward boundary of the auroral oval, auroral zone and sub-auroral latitudes. The objective of this paper is to study geomagnetic variations in the auroral region with a higherlatitude resolution than in Finch et al. (2008) to show that, even in the auroral region, there are significant variations in the geomagnetic disturbances and their dependence on different solar wind drivers, namely HSSs and CMEs.

\section{Data sources}

\subsection{IMAGE magnetometer data}

In this study, we used data from the IMAGE magnetometer network (Fig. 1), which is located in Fennoscandia and Svalbard (Viljanen and Häkkinen, 1997). The data set consists of 17 years of geomagnetic field measurements with a $10 \mathrm{~s}$ time resolution. The studied time period includes the years from 1994 to 2010 . The data are selected from magnetometer stations which have been operational since 1994. These stations and their corrected geomagnetic coordinates (CGMs) are listed in Table 1. CGMs were calculated for the year 2001 (altitude $0 \mathrm{~km}$ ) using the online service at http://omniweb.gsfc.nasa.gov/vitmo/cgm_vitmo.html.

We divided the magnetometer stations into three different groups based on their corrected geomagnetic latitudes and the typical behaviour of the magnetic disturbances. The frequency and intensity of the geomagnetic disturbances are dependent on the latitude because different ionospheric regions are connected to different parts of the magnetosphere.

The stations which belong to the magnetic latitude group 73-75 (geographic latitudes, Glat 76.5-78.9) are mostly located in the polar cap and mainly connected to open-lobe magnetic field lines. The stations which are located at magnetic latitudes $64-67^{\circ}$ (Glat 66.9-70.5) are connected to the 


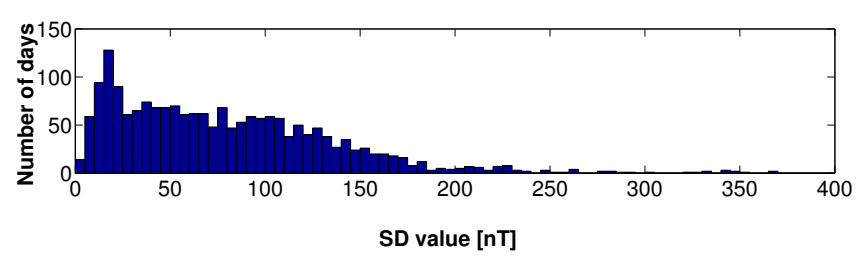

Figure 2. A typical yearly distribution of daily SD values of ground magnetic $X$ component for Region 2 in 2004. The median value of this distribution is $65 \mathrm{nT}$ and the mode is $15 \mathrm{nT}$.

plasma sheet most of the time. The magnetic latitudes 57$61^{\circ}$ (Glat 60.5-64.5) are connected to the almost dipolar field lines near the Earth. From north to south, we call these station groups Region 1 (the blue dots in Fig. 1), 2 (green dots) and 3 (yellow dots).

The conductivity in the ionosphere is largest in the auroral oval, which means that the strongest currents and magnetic field disturbances occur there. The average auroral electrojets are observed mainly between the geomagnetic latitudes $65^{\circ}$ to $70^{\circ} \mathrm{N}$ (Ritter et al., 2004; Juusola et al., 2009), which corresponds approximately to our Region 2. South and north of the average oval, geomagnetic variations are normally less frequent and much weaker. The auroral oval is not a stationary region, and it can expand towards the equator during intense storms and shrink towards the pole during quiet periods. Because of the moving of the oval, Regions 1 and 3 are affected by the currents flowing in the auroral zone.

\subsection{Solar wind and CME data}

We use solar wind speed and IMF measurements to describe the driver conditions in the solar wind. The solar wind data with 1-hour resolution have been propagated to the nose of the Earth's bow shock. The data have been downloaded from NASA Goddard Space Flight Center's OMNIWeb (http://omniweb.gsfc.nasa.gov/) and consist mainly of ACE (Advanced Composition Explorer) and Wind satellite measurements.

The CME event list is generated by CACTus (Computer Aided CME Tracking; Robbrecht et al., 2004). CACTus detects CMEs automatically in image sequences from LASCO on board the SOHO (Solar and Heliospheric Observatory) spacecraft. The listed events start in 1997, but the first full year of observations was in 2000. We use the list of all detected CMEs, although not all of them have been Earthdirected. The list of halo CMEs was also examined but resulted in a similar annual evolution, and, thus, we only show the occurrence of all CMEs as a proxy for the rate of Earthdirected CMEs.

\section{Results}

\subsection{Annual distributions of ground magnetic SDs}

The daily standard deviation of $10 \mathrm{~s}$ averages of the $X$ component (geographic north-south) of the ground magnetic field has been used as a measure of geomagnetic activity. We also analysed the behaviour of the other horizontal (eastwest, $Y$ ) magnetic field component and found it to be similar to that of the north-south component. Thus, we only show results of $B_{X}$. The SD values have been computed for each station and day separately. We use daily SD values as the main unit to average over different time sectors in order to study the average long-term behaviour. Yearly and monthly distributions of the SDs have been grouped into the three latitude regions based on visual inspection of the daily and yearly SD distributions. Within one latitude group the SD distributions of single stations are of a similar shape. The daily SD values range from 5 to $400 \mathrm{nT}$ but values above $100 \mathrm{nT}$ are a minority (8\% or all daily SDs in Region 1, $25 \%$ in Region 2 and $3 \%$ in Region 3). The SD values below $25 \mathrm{nT}$ are considered small, and they typically belong to the lowest $25 \%$ of daily values in the monthly SD distributions (not shown). The $25 \%$ quartile limit for the full data set is at about $15 \mathrm{nT}$, while for Regions 1, 2 and 3, it is at 26, 20 and 9nT, respectively. These values correspond to the quietest days of the month and, thus, mainly consist of the quiet-day variations. In Regions 1 and 2, SD values of 50-100 nT are common, while in Region 3 the average and median values are lower.

Figure 2 shows a yearly distribution of SDs in Region 2 in 2004. This shape is typical for Regions 1 and 2. Diurnal variations (Sq currents) are a likely cause for the maximum at the small SD values (typically at 10-30 nT), and the wide-spread peak at larger values is attributed to substorm and storm activity. When the number of substorms decreases, the number of large SDs also decreases. The histogram then becomes narrower and the small-value peak becomes the main feature of the distribution. Thus, SD distributions during geomagnetically quiet years are dominated by the peak at small values, while the second low wide-spread peak at larger values may even disappear. The distributions for active years maximise only at SDs larger than $40 \mathrm{nT}$.

Figures 3 and 4 show the annual distributions of the SD values in Regions 1 and 2 during the most extreme years of our data set: geomagnetically quiet (1997 and 2009) and active years (1994 and 2003). In both regions, the distributions of the most active years are clearly wider and extend to much larger SD values than during quiet years. In Region 1 (Fig. 3) during the quiet years, the majority of the SDs are less than $50 \mathrm{nT}$ and only a small fraction of values exceed $100 \mathrm{nT}$ ( $5.9 \%$ of the values in 1996 and only $0.3 \%$ of all values in 2009). In 2009, the SD values are even more strongly confined to below $50 \mathrm{nT}(88 \%)$ than during the previous minimum in 1997 (63\%). 


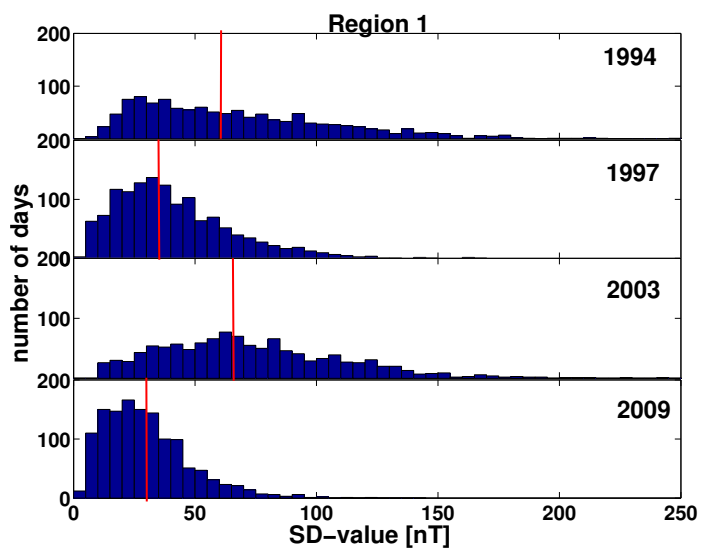

Figure 3. Yearly distributions of daily SD values of $B_{X}$ in Region 1 in 1994 and 2003 (active) and 1997 and 2009 (quiet). The red vertical lines show the median values of the distributions

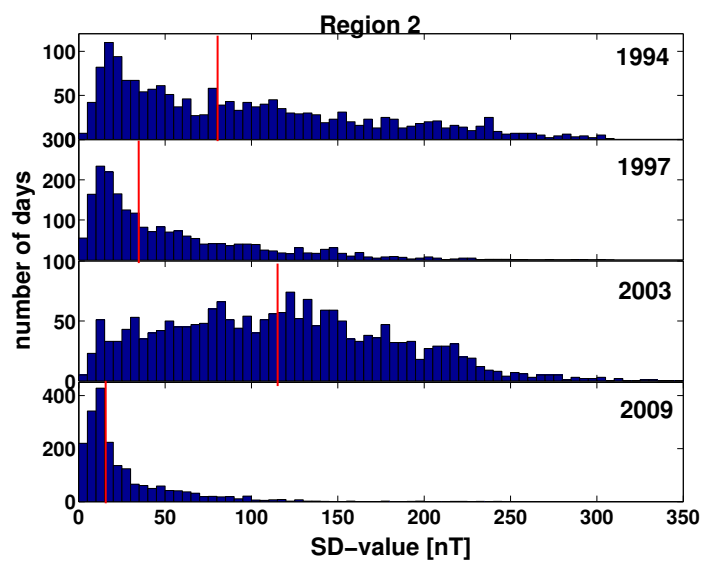

Figure 4. Yearly distributions of daily SD values of ground magnetic $X$ component in Region 2 during active (1994 and 2003) and quiet years (1997 and 2009). Note that the scale of $Y$ axis varies from year to year. The red vertical lines show the median values of the distributions

At Region 2 latitudes (Fig. 4), the difference between the 2 minimum years is much more pronounced than in Region 1. In Region 2 only a few SD values exceed $100 \mathrm{nT}(2.5 \%)$, with a distribution mode of $15 \mathrm{nT}$ in 2009. In 1997, on the other hand, the tail of SD values extends to about $250 \mathrm{nT}$. The distribution shape difference between the active years 1994 and 2003 is obvious: in 1994 the maximum occurs at values below $50 \mathrm{nT}$, and the distribution resembles the typical SD distribution in Fig. 2; in 2003 the Sq variations at small $\mathrm{SD}$ values are not visible at all, and the distribution peaks at values larger $100 \mathrm{nT}$ with the mode of $125 \mathrm{nT}$.

\subsection{Solar wind effect on geomagnetic disturbance level}

The time series of monthly-averaged SDs have been smoothed using a 13-month running mean. We set half weights for the months at the start and end of the time series.

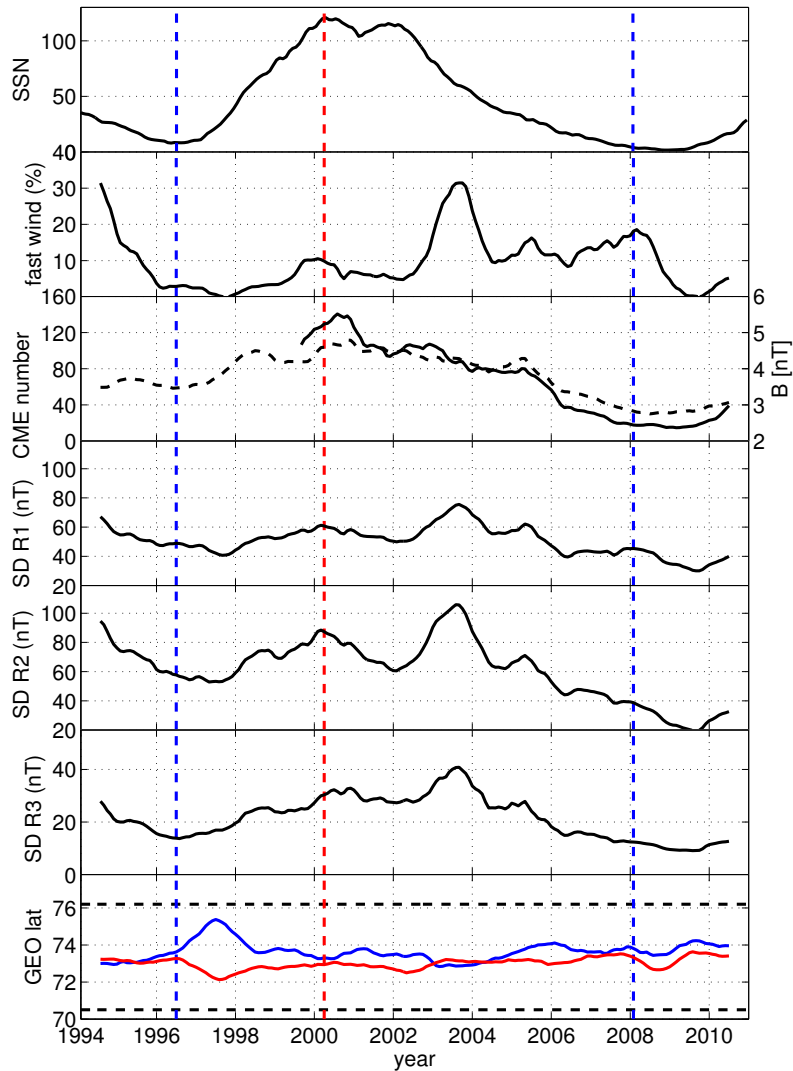

Figure 5. From top to bottom: number of sunspots, the percentage of the time when solar wind speed value exceeds $600 \mathrm{~km} \mathrm{~s}^{-1}$, the number of CMEs (solid) together with the IMF magnitude (dashed line), SD values in Region 1, SD values in Region 2, SD values in Region 3 in 1994-2010, and the maximum geographic latitudes of the maximum eastward (blue) and westward (red) electrojet current densities in degrees. All the values are 13-month-smoothed monthly averages. The black dashed horizontal lines in the bottom panel show the southern boundary of Region 1 (upper line) and the northern boundary of Region 2 (bottom line). The blue dashed lines shows the starting and ending point of solar cycle 23 and the red dashed line show the month of the smoothed maximum sunspot number.

The same smoothing method is often used for the sunspot number. The first and last six monthly mean values have been removed from the smoothed curves in Fig. 5. On average the largest geomagnetic variations occur in Region 2, as illustrated by the previous figures, although some single high SD values can be found in Region 3 during very intense storms. (The largest Region 3 values are not visible in the monthly average curves but can be seen in the annual distributions, not shown in this paper).

In addition to the ground magnetic SD values, Fig. 5 shows the monthly sunspot number (top panel), the monthly occurrence rate of high-speed solar wind (second panel), the monthly number of CMEs together with the IMF magnitude (third panel), and the geographic latitudes of the maximum 
eastward (blue curve) and westward (red curve) electrojet current densities in degrees (bottom panel). All monthly values have been smoothed using the 13-month moving average technique. The sunspot number (top panel) defines the solar cycle. The maximum of sunspot cycle 23 consisted of 2 local peaks in 2000 and 2001, and the minimum was reached in 2009. The blue dashed vertical lines show the starting and ending point of solar cycle 23 and the red dashed line shows the month of the maximum sunspot number during the cycle.

High-speed solar wind (second panel) has been defined as time periods for which the solar wind speed exceeds $600 \mathrm{~km} \mathrm{~s}^{-1}$. The monthly total duration of the high-speed solar wind has been divided by the total duration of each month $(\%)$. We use this occurrence rate of high-speed solar wind as a proxy for the high-speed stream (HSS) occurrence. This assumption is justified by comparing our results to the ones by Baumann (2008), who used similar threshold values (from 600 to $700 \mathrm{~km} \mathrm{~s}^{-1}$ ) as a definition of HSS and got a qualitatively similar annual distribution (their figures 27 and 28).

The IMF magnitude, plotted together with the number of CMEs (third panel), agrees very well with CME activity since 2000. Thus, IMF could be used as a proxy for the CME occurrence rate for the earlier years where observations of the Sun's disk were not yet available. IMF components have also been examined but not included here since they evolved similarly to the total field.

The latitudes of the maximum electrojet current densities (bottom panel) have been defined by computing the ionospheric equivalent currents using the spherical elementary current system method (Amm, 1997; Amm and Viljanen, 1999) from the IMAGE 1-minute data. The average latitude of the maximum electrojets reflects the location of the auroral oval during the studied time period. On average the maximum electrojet currents flow between our latitude Regions 1 and 2. The magnetometer network is sparse there because it is above the ocean. The width of the electrojet currents is of the order of a few degrees (Juusola et al., 2009), which means that the southern boundary of the auroral oval is typically above Fennoscandia and the northern boundary regularly flows above the Svalbard stations.

The decrease (starting in 2005) in monthly mean SD values before the latest sunspot minimum (in 2008, blue line in Fig. 5) was not as monotonic in Region 1 as it was in the other regions. For example, the year 2008, when the solar wind speed was still rather high, stands out as having geomagnetic activity that appears levelled out when compared to previous years. The minimum of sunspot cycle 22 occurred at the very end of 1997 in Region 1, while at Region 2 latitudes, the whole year 1997 was geomagnetically quiet. In Region 3, the minimum was reached already in 1996, suggesting that the storm activity ceased earlier than the smaller events within Region 2. Furthermore, a local SD maximum was seen in Regions 2 and 3 in 2000. Similar peaks cannot be observed in Region 1, where the mean values of 2000 do not differ clearly from adjacent years.
In 1994 and 2003, fast solar wind (second panel) occurred exceptionally often as compared to the rest of the time series. The difference between the HSS occurrence rate between those 2 years was negligible. The mean SD peaks during the same years suggest that geomagnetic activity was enhanced due to HSS activity. During the highest geomagnetic activity level, in 2003, the CME rate did not experience an exceptionally high value. The highest CME occurrence rate was observed during 2000, but the ground magnetic response was only a mild increase of SDs, clearest at Region 3 stations.

In 2006-2008 the CME occurrence decayed. During those years also the mean SD values decreased steadily. The decrease was most prominent in Regions 2 and 3 and changed less in Region 1. Local enhancements are probably caused by high-speed solar wind streams, since the HSS rate stayed at a relatively high level until the end of 2008. The SD minima in Regions 1 and 2 correlated best with the occurrence of HSS, while Region 3 SD reached its minima at the same time as the IMF. In 2009, the occurrences of HSSs, CMEs and high values of monthly SDs of all latitude groups reached a minimum. Comparing the monthly mean SD values during the minima of sunspot cycles 22 and 23, it is evident that the values were lower at the minimum of cycle 23 . In particular, SD values in Region 2 during the sunspot minimum 23 were about half those observed during the minimum of cycle 22 . The low occurrence rates of high-speed solar wind was very similar between the 2 minimum years, while the IMF was much weaker in 2009 than in 1997. Finally, during 2010, the HSS and CME occurrence (second and third panel) began to increase from the minimum values. The same kind of enhancement can also be seen in the ground magnetic field.

\section{Discussion}

We have studied the ground magnetic field variations during a 17-year time period and analysed their distributions in three different latitude regimes. We examined monthly SD values of the ground $B_{X}$, which gives the range of variations in the $\boldsymbol{B}$ field. We have further compared the changes in geomagnetic activity level to solar wind driving. We have used raw magnetic field data, which include the baseline, diurnal and seasonal variations. These variations can be locally and temporally large (Watermann et al., 2011) and cannot be neglected in geomagnetic event analysis. However, averaging over full years to look at the long-term evolution of daily and monthly SDs, we consider this a minor issue. It has also been pointed out by Watermann et al. (2011) that the quiet-time variations in the vertical magnetic field component are much more severe than the horizontal ones. The average auroral oval region (Region 2 in this study) maps to the magnetospheric plasma sheet, which is prone to dynamic processes. Thus, geomagnetic disturbances are also largest in Region 2 due to the large conductivity and electric currents in the oval. Region 1 disturbances are always smaller, and Region 3 vari- 
ations are the smallest ones most of the time, except during strong magnetic storms. These results agree with the latitude behaviour of the time derivatives of the horizontal geomagnetic field reported by Watermann and Gleisner (2009). The years 2008-2010 were exceptional in our study. The largest variations occurred in Region 1. This is due to the shrunk oval during exceptionally weak solar wind driving, as described by Pulkkinen et al. (2011). The contraction of the oval can be also seen in the bottom panel in Fig. 5 when the average location of the maximum electrojet currents moved towards higher latitudes in 2009-2010.

According to Tsurutani et al. (2011), the Ap index, which is a range index compiled from 11 to 13 stations in the midlatitude Northern Hemisphere (Rostoker, 1972), also had exceptional values in 2003 and 2009. The Ap index values for the minimum of sunspot cycle 23 were considerably lower than for the minimum of cycle 22 .

The variations in the monthly SD levels mainly follow the occurrence of high-speed solar wind. The clearest difference occurred in 2006-2009: the monthly SD values fall steadily, but the occurrence of high-speed wind stays elevated until the second half of 2008. In contrast, the monthly SD values follow the decline of the CME rate and the IMF magnitude in 2006-2009. Apart from this difference, the strongest geomagnetic activity (the largest SD values) in every latitude region coincided with a high occurrence rate of high-speed solar wind, in agreement with the results of Tanskanen et al. (2011). The fast solar wind occurrence peaked in 2003, while the CME rate had already started to decrease from its highest level. Similarly, the high-speed solar wind was frequent during strong geomagnetic activity in 1994, while the CME rate (estimated from the IMF magnitude) was at an even lower level in 1994 than in 2003. CME activity peaked in 2000, causing only a small increase in the monthly SDs.

CME activity has the strongest effect on the behaviour of the monthly SDs in Region 3 and the weakest in Region 1. Moderate-to-intense storms as the main driver of the magnetic disturbances at Region 3 latitudes have been reported by earlier studies (e.g. Gleisner et al., 2006). The local bump in monthly SDs during 2000-2001 correlates with the peak in the CME occurrence. The other latitude regions also show elevated monthly SD values in early 2000 but these occurred before the CME maximum and instead coincided with the small increase in the HSS rate in late 1999 and early 2000. The monthly SD evolution in Region 3 follows the decrease of the CME rate in 2005-2009 more precisely than the SDs in the other regions. The geomagnetic minimum during sunspot cycle 22 occurred in 1996, at the same time as the IMF minimum, while the minimum in other latitude regions was reached simultaneously with the HSS minimum.

Even if the solar-wind-magnetosphere coupling is often studied using the coupling functions which combine $\boldsymbol{B}$ and $V$, we compare the geomagnetic activity, the solar wind speed, the number of CMEs and IMF magnitude separately with the geomagnetic variations. This makes it easier to see the impact of each solar wind driver on the monthly SDs. The problem which would arise from using a coupling function is that such functions are superpositions of multiple solar wind drivers whose responses are latitude-dependent. Because we are not using well-known geomagnetic indices and we are studying narrower latitude bands than the previous studies, it would not be straightforward to choose the best coupling function.

Numerous studies have examined the correspondence between different geomagnetic indices and solar activity. For example, aa and Ap indices have been found to correlate with the sunspot number with roughly a 2-year time lag (Echer et al., 2004; Verbanac et al., 2011). A good correspondence between the aa index and the number of days per year with a solar wind speed higher than $500 \mathrm{~km} \mathrm{~s}^{-1}$ has been reported (Echer et al., 2004). Verbanac et al. (2011) noted that the evolution of the Ap and Dst (sensitive to storm-time disturbances) indices correlates best with the interplanetary magnetic field (IMF) variations, which strongly affect the ring current evolution (Echer et al., 2008).

The auroral electrojet (AE; Rostoker, 1972) index activity follows the HSS occurrence rate well, and it primarily detects magnetospheric substorm activity. This indicates that the geomagnetic activity in Regions 1 and 2 is more clearly associated with the substorms than the storm-driven activity in Region 3.

Tsurutani et al. (2011) studied the minima in geomagnetic activity using the Ap index during sunspot cycles 22 and 23 and identified their time of occurrence relative to the sunspot minima. The Ap index showed exceptional values in 2003 and 2009, and its minimum in sunspot cycle 23 was considerably lower than the minimum during sunspot cycle 22 .

The declining IMF in 2006-2010 has been related to the weakening solar flux (Owens et al., 2008) and the decay of the solar magnetic field (Tsurutani et al., 2011). The exceptionally low solar magnetic field, the disappearance of equatorial and low-latitude coronal holes and the appearance of midlatitude coronal holes on the Sun were the most important factors in causing the geomagnetic activity minimum in 2009.

On average, the geomagnetic activity cycle follows the sunspot cycle with a delay of 1-2 years (Nevanlinna and Pulkkinen, 2001), which may be true for global indices, such as aa and Ap. At different latitude regions, the delay time may vary. Our results from three latitude bands show significant temporal variations between the regions.

\section{Conclusions}

In this paper, we have studied geomagnetic variations in three different high-latitude regions in 1994-2010 as measured by the daily and monthly standard deviation values of the geomagnetic field. On average, the largest geomagnetic variations occur in Region 2, which is located mainly within the 
auroral oval, and the weakest variations occur in Region 3 (sub-auroral latitudes). The SD values in Regions 1 and 2 were found to correlate best with the occurrence rate of the high-speed solar wind and substorm activity, while Region 3 is affected the most by CME activity.

The year 2003 was geomagnetically very active in all three regions due to the high rate of high-speed solar wind. The deep and long quiet period (2005-2009) was followed by an activity increase in 2010, but it was still very weak compared to the previous rising phase in 1997-1998. This geomagnetically quiet period correlated best with the weakening of the interplanetary magnetic field and solar magnetic flux. The deep and long geomagnetic activity minimum during sunspot cycle 23 was most clearly visible in Region 2 and was weakest in Region 1. In Region 3 the geomagnetic activity minimum during cycle 23 was longer and more quiet than usual.

When using geomagnetic activity indices to describe the solar cycle evolution of the global disturbance level, it should be noted that the effect of the different solar wind drivers on the geomagnetic activity is strongly dependent on latitude.

Acknowledgements. We thank all institutes maintaining the IMAGE magnetometer network, and we acknowledge use of NASA/GSFC's Space Physics Data Facility's OMNIWeb and OMNI data. Special thanks to Ari Viljanen for providing the electrojet data. The SOHO/LASCO data used here are produced by a consortium of the Naval Research Laboratory (USA), Max-Planck-Institut für Aeronomie (Germany), Laboratoire d'Astronomie (France) and the University of Birmingham (UK). SOHO is the result of international cooperation between ESA and NASA. M. Myllys acknowledges Academy of Finland project 1267087.

The topical editor S. Milan thanks M. Lockwood and one anonymous referee for help in evaluating this paper.

\section{References}

Amm, O.: Ionospheric elementary current systems in spherical coordinates and their application, J. Geomagn. Geoelectr., 49, 7, 947-955, 1997.

Amm, O. and Viljanen, A.: Ionospheric disturbance magnetic field continuation from the ground to the ionosphere using spherical elementary current systems, Earth Planets Space, 51, 6, 431-440, 1999.

Akasofu, S.-I.: Energy coupling between the solar wind and the magnetosphere, Space Sci. Rev., 28, 2, 121-190, 1981.

Baumann, G.: High-Speed Structures and Interaction with Magnetosphere, Master's thesis, the Institute of Theoretical Physics, University of Zürich, Switzerland, http://www.physikstudium.uzh.ch/fileadmin/physikstudium/ Masterarbeiten/Baumann_2008.pdf, 2008.

Beamish, D., Clark, T., Clarke, E., and Thomson, A.: Geomagnetically induced currents in the UK: geomagnetic variations and surface electric fields, J. Atmos. Sol.-Terr. Phy., 64, 1779-1792, 2002 .
Brueckner, G. E., Howard, R. A., Koomen, M. J., Korendyke, C. M., Michels, D. J., Moses, J. D., Socker, D. G., Dere, K. P., Lamy, P. L., and Llebaria, A.: The large angle spectroscopic coronagraph (LASCO), Sol. Phys., 162, 357-402, 1995.

Echer, E., Gonzalez, W. D., Tsurutani, B. T., and Gonzalez, A. L. C.: Interplanetary conditions causing intense geomagnetic storms (Dst $<-100 \mathrm{nT}$ ) during solar cycle 23 (1996-2006), J. Geophys. Res.-Space (1978-2012), 113, A5, 2008.

Echer, E., Gonzalez, W. D., Gonzalez, A. L. C., Prestes, A., Vieira, L. E. A., Dal Lago, A., Guarnieri, F. L., and Schuch, N. J.: Longterm correlation between solar and geomagnetic activity, J. Atmos. Sol.-Terr. Phy., 66, 1019-1025, 2004.

Eddy, J. A.: The maunder minimum, Science, 192, 4245, 1189 1202, doi:10.1126/science.192.4245.1189, 1976.

Falayi, E. O. and Rabiu, B. A.: Dependence of time derivative of horizontal geomagnetic field on sunspot number and aa index, Acta Geophys., 61, 211-222, 2013.

Finch, I. and Lockwood, M.: Solar wind-magnetosphere coupling functions on timescales of 1 day to 1 year, Ann. Geophys., 25, 495-506, doi:10.5194/angeo-25-495-2007, 2007.

Finch, I. D., Lockwood, M. L., and Rouillard, A. P.: Effects of solar wind magnetosphere coupling recorded at different geomagnetic latitudes: Separation of directly-driven and storage/release systems, Geophys. Res. Lett., 35, L21105, doi:10.1029/2008GL035399, 2008.

Fraser-Smith, A. C.: Spectrum of the geomagnetic activity index Ap, J. Geophys. Res., 77, 4209-4220, 1972.

Gleisner, H., Rasmussen, O., and Watermann, J.: Large-magnitude geomagnetic disturbances in the North Sea region: Statistics, causes, and forecasting, Adv. Space Res., 37, 1169-1174, doi:10.1016/j.asr.2005.04.082, 2006.

Gonzalez, W. D., Gonzalez, A. L. C., and Tsurutani, B. T.: Dualpeak solar cycle distribution of intense geomagnetic storms, Planet. Space Sci., 38, 181-187, 1990.

Gopalswamy, N., Nunes, S., and Howard, R.: Variability of solar eruptions during cycle 23, Adv. Space Res., 34, 391-396, 2004.

Imber, S. M., Milan, S. E. and Lester, M.: Solar cycle variations in polar cap area measured by the superDARN radars, J. Geophys. Res.-Space, 118, 6188-6196, 2013.

Juusola, L., Kauristie, K., Amm, O., and Ritter, P.: Statistical dependence of auroral ionospheric currents on solar wind and geomagnetic parameters from 5 years of CHAMP satellite data, Ann. Geophys., 27, 1005-1017, doi:10.5194/angeo-27-10052009, 2009.

Juusola, L., Østgaard, N., Tanskanen, E., Partamies, N., and Snekvik, K.:, Earthward plasma sheet flows during substorm phases, J. Geophys. Res., 116, A10228, doi:10.1029/2011JA016852, 2011.

Lockwood, M., Barnard, L., Nevanlinna, H., Owens, M. J., Harrison, R. G., Rouillard, A. P., and Davis, C. J.: Reconstruction of geomagnetic activity and near-Earth interplanetary conditions over the past $167 \mathrm{yr}$ - Part 1: A new geomagnetic data composite, Ann. Geophys., 31, 1957-1977, doi:10.5194/angeo-31-19572013, 2013a.

Lockwood, M., Barnard, L., Nevanlinna, H., Owens, M. J., Harrison, R. G., Rouillard, A. P., and Davis, C. J.: Reconstruction of geomagnetic activity and near-Earth interplanetary conditions over the past $167 \mathrm{yr}-$ Part 2: A new reconstruction of the 
interplanetary magnetic field, Ann. Geophys., 31, 1979-1992, doi:10.5194/angeo-31-1979-2013, 2013b.

Mayaud, P. N.: The aa indices: A 100-year series characterizing the magnetic activity, J. Geophys. Res., 77, 6870-6874, 1972.

Meng, C. I.: Dynamic variation of the auroral oval during intense magnetic storms, J. Geophys. Res., 89, 227-235, 1984.

Mittal, N. and Narain, U.: On some properties of coronal mass ejections in solar cycle 23, New Astron., 14, 341-346, 2009.

Nevanlinna, H. and Pulkkinen, T. I.: Auroral observations in Finland: Results from all-sky cameras, 1973-1997, J. Geophys. Res.-Space (1978-2012), 106, 8109-8118, 2001.

Newell, P. T., Sotirelis, T., Liou, K., and Rich, F. J.: Pairs of solar wind-magnetosphere coupling functions: Combining a merging term with a viscous term works best, J. Geophys. Res.-Space (1978-2012), 113, A4, 2008.

Owens, M., Arge, C., Crooker, N., Schwadron, N., and Horbury, T.: Estimating total heliospheric magnetic flux from single-point in situ measurements, J. Geophys. Res.-Space (1978-2012), 113, A12103, doi:10.1029/2008JA013677, 2008.

Partamies, N., Juusola, L., Tanskanen, E., and Kauristie, K.: Statistical properties of substorms during different storm and solar cycle phases, Ann. Geophys., 31, 349-358, doi:10.5194/angeo31-349-2013, 2013.

Pulkkinen, T. I., Tanskanen, E., Viljanen, A., Partamies, N., and Kauristie, K.: Auroral electrojets during deep solar minimum at the end of solar cycle 23, J. Geophys. Res., 116, A04207, doi:10.1029/2010JA016098, 2011.

Richardson, I. G., Cliver, E. W., and Cane, H. V.: Sources of geomagnetic storms for solar minimum and maximum conditions during 1972-2000, Geophys. Res. Lett., 28, 2569-2572, 2001.

Ritter, P., Lühr, H., Viljanen, A., Amm, O., Pulkkinen, A., and Sillanpää, I.: Ionospheric currents estimated simultaneously from CHAMP satelliteand IMAGE ground-based magnetic field measurements: a statisticalstudy at auroral latitudes, Ann. Geophys., 22, 417-430, doi:10.5194/angeo-22-417-2004, 2004.

Robbrecht, E. and Berghmans, D.: Automated recognition of coronal mass ejections (CMEs) in near-real-time data, Astron. Astrophys., 425, 1097-1106, 2004.

Rostoker, G.: Geomagnetic indices, Rev. Geophys., 10, 935-950, 1972.

Schove, D. J.: The sunspot cycle, 649 BC to AD 2000, J. Geophys. Res., 60, 127-146, 1955.

Solovyev, S. I., Boroyev, R. N., Moiseyev, A. V., Du, A., and Yumoto, K.: Dynamics of the ionospheric electric currents and auroral luminosity boundaries during strong magnetic storms, Geomagn. Aeronomy, 49, 450-460, 2009.
Tanskanen, E., Slavin, J., Tanskanen, A., Viljanen, A., Pulkkinen, T., Koskinen, H., Pulkkinen, A., and Eastwood, J.: Magnetospheric substorms are strongly modulated by interplanetary high-speed streams, Geophys. Res. Lett., 32, L16104, doi:10.1029/2005GL023318, 2005.

Tanskanen, E., Pulkkinen, T., Viljanen, A., Mursula, K., Partamies, N., and Slavin, J.: From space weather toward space climate time scales: Substorm analysis from 1993 to 2008, J. Geophys. Res.Space (1978-2012), 116, A00I34, doi:10.1029/2010JA015788, 2011.

Tenfjord, P. and Østgaard, N.: Energy transfer and flow in the solar wind-magnetosphere-ionosphere system: A new coupling function, J. Geophys. Res.-Space, 118, 5659-5672, 2013.

Tsurutani, B. T., Echer, E., and Gonzalez, W. D.: The solar and interplanetary causes of the recent minimum in geomagnetic activity (MGA23): a combination of midlatitude small coronal holes, low IMF $\boldsymbol{B}_{Z}$ variances, low solar wind speeds and low solar magnetic fields, Ann. Geophys., 29, 839-849, doi:10.5194/angeo-29-839-2011, 2011.

Verbanac, G., Mandea, M., Vršnak, B., and Sentic, S.: Evolution of solar and geomagnetic activity indices, and their relationship: 1960-2001, Sol. Phys., 271, 183-195, 2011.

Vickrey, J. F., Vondrak, R. R, and Matthews, S. J.: The diurnal and latitudinal variation of auroral zone ionospheric conductivity, J. Geophys. Res.-Space (1978-2012), 86, 65-75, 1981.

Viljanen, A. and Häkkinen, L.: IMAGE magnetometer network, Satellite-ground based coordination sourcebook, 1198, 111, 1997.

Viljanen, A., Nevanlinna, H., Pajunpää, K., and Pulkkinen, A.: Time derivative of the horizontal geomagnetic field as an activity indicator, Ann. Geophys., 19, 1107-1118, doi:10.5194/angeo-191107-2001, 2001.

Watermann, J. and Gleisner, H.: Geomagnetic variations and their time derivatives during geomagnetic storms at different levels of intensity, Acta Geophys., 57, 197-208, 2009.

Watermann, J., Gleisner, H., and, Rasmussen, T. M.: A geomagnetic activity forecast for improving the efficiency of aeromagnetic surveys in Greenland, Adv. Space Res., 47, 2172-2181, doi:10.1016/j.asr.2010.02.005, 2011.

Wolf, R. A.: Ionosphere-magnetosphere coupling, Space Sci. Rev., 17, 537-562, 1975. 